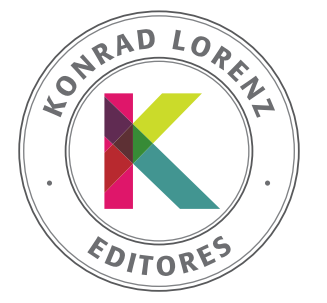

SUMA PSICOLÓGICA

http://editorial.konradlorenz.edu.co/suma-psicologica.html

\title{
Proceso de toma de decisiones bajo incertidumbre y bajo riesgo en niños y adolescentes con Trastorno por Déficit de Atención e Hiperactividad
}

\author{
Josefina Rubiales, Micaela Reyna, Liliana Bakker y Sebastián Urquijo \\ Instituto de Psicología Básica, Aplicada y Tecnología (IPSIBAT), Consejo Nacional de Investigaciones Científicas y Técnicas (CONICET), \\ Facultad de Psicología, Universidad Nacional de Mar del Plata, Argentina
}

Recibido el 4 de abril de 2019; aceptado el 30 de julio de 2019

PALABRAS CLAVE

Toma de decisiones, niños, adolescentes, TDAH

\section{KEYWORDS}

Decision Making, children, adolescents, ADHD

\begin{abstract}
Resumen El objetivo fue analizar y comparar el proceso de toma de decisiones bajo riesgo y bajo incertidumbre y el nivel de conciencia respecto de cuáles son las opciones ventajosas y desventajosas en niños y adolescentes con y sin Trastorno por Déficit de Atención e Hiperactividad (TDAH). Se realizó un estudio descriptivo comparativo con un diseño de tipo ex post facto retrospectivo con dos grupos, uno de cuasi control. La muestra clínica estuvo compuesta 35 niños y adolescentes con diagnóstico de TDAH y la muestra control por 35 niños y adolescentes sin el diagnóstico, divididas ambas muestras en dos grupos etarios. Los resultados evidenciaron que los adolescentes con TDAH tienen un comportamiento más riesgoso a pesar de tener conciencia sobre el nivel de riesgo implicado, mientras que los niños con TDAH tienen un comportamiento similar en la toma de decisiones que los niños sin este diagnóstico, aunque presentan un menor nivel de conciencia respecto de cuáles son las opciones ventajosas y desventajosas. No se encontraron diferencias en términos de resultados obtenidos, por lo que el mayor nivel de riesgo asumido por adolescentes con TDAH parece ser más compatible con un estilo diferente en la toma de decisiones, que con una propensión a la toma de decisiones desfavorable.

(c) 2019 Fundación Universitaria Konrad Lorenz. Este es un artículo Open Access bajo la licencia CC BY-NC-ND (http://creativecommons.org/licenses/bync-nd/4.0/).
\end{abstract}

Decision-making process under risk and under uncertainty in children and adolescents with Attention-Deficit/Hyperactivity Disorder

\begin{abstract}
The aim was to analyze and compare the decision-making process under risk and under uncertainty and the level of awareness regarding which are the advantageous and disadvantageous options in children and adolescents with and without Attention-Deficit/Hyperactivity Disorder (ADHD). A comparative descriptive study was proposed with a retrospective ex post facto design with two groups, one of quasi control. The clinical sample consisted of 35 children and adolescents with a diagnosis of ADHD and the control sample by 35 children and adolescents without a diagnosis divided both samples into two age groups. The results showed
\end{abstract}

* Autor para correspondencia.

Correo electrónico: josefinarubiales@gmail.com 
that adolescents with ADHD have a riskier behavior despite being aware of the level of risk involved, while children with ADHD have similar behavior in decision making than children without this diagnosis, although they present a lower level of conscience regarding what are the advantageous and disadvantageous options. No differences were found in terms of results obtained, so the higher level of risk assumed in decision making by adolescents with ADHD seems to be more compatible with a different style of decision making, than with a propensity to take unfavorable decisions.

(c) 2019 Fundación Universitaria Konrad Lorenz. This is an open access article under the CC BYNC-ND license (http://creativecommons.org/licenses/bync-nd/4.0/).

El Trastorno por déficit de atención con hiperactividad (TDAH) es definido como un patrón persistente de síntomas de inatención, hiperactividad e impulsividad, que se presenta con mayor frecuencia e intensidad que en las personas con un grado de desarrollo similar (APA, 2013). Es considerado uno de los trastornos del neurodesarrollo más frecuentes en la población infantil (Mariño et al., 2018), con una prevalencia mundial del 5.9 a $7.1 \%$ en niños y adolescentes (Willcutt, 2012), y específicamente en Argentina del 4\% (Grañana, 2017).

Diversos estudios refieren que los niños y adolescentes con TDAH son más propensos a presentar dificultades en la toma de decisiones (TD), ya que tienden a ser impulsivos, participan en actividades más riesgosas, corren riesgos innecesarios, descuidan las consecuencias futuras y son más propensos a accidentes (Groen, Gaastra, Lewis-Evans, \& Tucha, 2013).

La TD es un proceso definido como la elección de una opción entre un conjunto de alternativas existentes, considerando los posibles resultados de las selecciones y sus consecuencias en el comportamiento presente y futuro (Kahneman, \& Tversky, 1984). La capacidad de diferenciar entre elecciones ventajosas y desventajosas aumenta con la edad. Schiebener y Brand (2015) señalan que los niños no logran anticipar los resultados de sus decisiones porque la habilidad de realizar elecciones ventajosas está en desarrollo. En relación con los adolescentes, si bien existen resultados controvertidos, diversos estudios coindicen en señalar que muestran una capacidad de TD comparable a la de los adultos (Reyna, \& Farley, 2006; Van Duijvenvoorde, 2013).

Las investigaciones sobre la TD en niños y adolescentes con TDAH reportan resultados controvertidos (Rubiales, Urquijo, Said, \& Macbeth, 2017). Algunos estudios aportan evidencias de que parecerían más propensos a arriesgarse o a tomar decisiones desfavorables (Drechsler, Rizzo, \& Steinhausen, 2010; Kroyzer, Gross-Tsur, \& Pollak, 2014; Patros et al., 2015). En esta línea, el modelo dual de Sonuga-Barke (2002) sostiene que las personas con TDAH presentan una alteración en los circuitos de recompensa que provoca que la señal de recompensa demorada no sea detectada, por lo cual prefieren recompensas inmediatas aunque pequeñas, antes que recompensas mayores pero demoradas en el tiempo (Wilbertz et al., 2013). Sin embargo, otras investigaciones reportan no haber encontrado evidencias suficientes para sustentar estas afirmaciones (Garon, Moore, \& Waschbusch, 2006; Humphreys, Tottenham, \& Lee, 2018; Scheres et al., 2006). Asimismo, resulta interesante señalar que no se han encontrado estudios de comparación de ambos tipos de TD en población con TDAH (Rubiales et al., 2017).

Los estudios de TD en población infanto-juvenil con TDAH no siempre han analizado el desempeño de niños y adolescentes como grupos etarios diferenciados (Hovik et al., 2015; Lambek et al., 2011; Masunami, Okazaki, \& Maekawa, 2009; Scheres et al., 2006; Skogli et al., 2014; Skogli,
Teicher, Andersen, Hovik, \& Oie 2013). Sin embargo, los estudios que analizaron el desempeño en adolescentes exclusivamente (Kroyzer et al., 2014; Pollak, \& Shoham, 2015; Toplak, Jain, \& Tannok, 2005), encontraron evidencias de un desempeño inferior de los jóvenes con TDAH. En este sentido, Bernal, Montaña, Acosta y Rojas (2015), describen que en los adolescentes con TDAH no se observa una mejora gradual en el desempeño de la TD posiblemente debido a un aumento de la búsqueda de sensaciones y a la tendencia a alejarse de la norma y sobreestimar los peligros, lo cual se ve reflejado en la adopción de comportamientos de riesgo (Reyna, \& Farley, 2006).

Respecto a las investigaciones neuropsicológicas acerca de la TD, los autores coinciden en diferenciar entre la TD bajo incertidumbre (TDBI) y la TD bajo riesgo (TDBR) (Bechara, Damasio, Damasio, \& Anderson, 1994; Brand, Labudda, \& Markowitsch, 2006). El proceso de TD bajo incertidumbre (TDBI) se presenta cuando la persona no tiene información sobre los posibles resultados y la probabilidad de las consecuencias, por lo que debe descubrir las reglas para resolver el problema utilizando la retroalimentación que obtiene como resultado de sus elecciones, decidiendo intuitivamente (Bechara, 2004; Brand, Heinze, Labudda, \& Markowitsch, 2008). En cambio, en la TD bajo riesgo (TDBR) existe información explícita sobre las consecuencias potenciales y las probabilidades de recompensas o castigos, por lo tanto, las decisiones se realizan a partir de los conocimientos disponibles sobre la situación (Liebherr, Schiebener, Averbeck, \& Brand, 2017), estando estrechamente asociada a las funciones ejecutivas (Brand et al., 2008).

Específicamente respecto a la TDBI, diversos estudios han reportado diferencias significativas entre población infanto-juvenil con y sin TDAH (Drechsler et al., 2010; Geurts, Van der Oord, \& Crone, 2006; Hobson, Scott, \& Rubia, 2011; Toplak et al., 2005) y han evidenciado que los niños y adolescentes con TDAH toman decisiones más riesgosas. Patros et al. (2015) hallaron que los niños con TDAH responden más impulsivamente durante una tarea de dos opciones, pero no durante una tarea de cinco opciones, sustentando la idea de que la impulsividad relacionada con el TDAH no es omnipresente, sino que depende de la variación de la demanda y/o el contexto. Por el contrario, otros estudios no han encontrado evidencias de diferencias significativas en el desempeño en TDBI en esta población clínica (Antonini, Becker, Tamm, \& Epstein, 2015; Geurts et al., 2006; Hovik, \& Hansen, 2015; Lambek et al., 2011; Masunami et al., 2009; Oie, Skogli, Andersen, Hovik, \& Hugdahl, 2014).

En relación con la TDBR, Kroyzer et al. (2014) informan que los adolescentes con TDAH realizan apuestas menores y eligen la opción menos probable con más frecuencia que el grupo control, pero presentan la misma velocidad al tomar decisiones. Por el contrario, Scheres et al. (2006) no encontraron diferencias significativas, lo cual, según estos autores, se explicaría por una mayor motivación en los adolescentes con TDAH ante la expectativa de obtener gran recompensa, lo que facilitaría la espera. 
Estos resultados controvertidos podrían ser explicados por diferentes factores, tales como el tamaño y la composición de la muestra, los instrumentos y las tareas utilizadas, la presencia de comorbilidad y el uso de medicación (Rubiales et al., 2017). Abordar el estudio del desarrollo de la TD en niños y adolescentes con TDAH podría ser útil en la identificación de dificultades y potencialidades, aportando información relevante a la conceptualización del trastorno para profundizar el modelo de evaluación y diseñar tratamientos acordes a las necesidades particulares de esta población, que permitan mejorar su calidad de vida.

Por este motivo, se propuso como objetivo analizar y comparar el proceso de TDBR y TDBI en niños y adolescentes con y sin TDAH. Asimismo, se planteó analizar y comparar el nivel de conciencia o autopercepción en ambos grupos, respecto de cuáles son las opciones ventajosas y desventajosas.

\section{Método}

\section{Tipo de estudio}

Se corresponde con un estudio descriptivo comparativo con un diseño de tipo ex post facto retrospectivo con dos grupos, uno de cuasi control, según la clasificación de Montero y León (2007).

\section{Participantes}

La muestra se planeó de forma intencional con 70 niños escolarizados de la ciudad de Mar del Plata, Argentina, divididos en dos grupos, seleccionados por muestreo no probabilístico por conveniencia. La muestra clínica se conformó por 35 niños con diagnóstico de TDAH derivados por médicos neurólogos de instituciones de salud de la ciudad, 29 niños y seis niñas, con edades comprendidas entre ocho y 16 años $(M=10.71 ; D E=2.00)$, divididos en dos grupos etarios, 15 niños de ocho a 11 años y 20 adolescentes de 12 a 16 años. La muestra control, estuvo compuesta por 35 niños sin diagnóstico de TDAH emparejados por sexo, edad y nivel de instrucción. Los criterios de inclusión para la muestra clínica fueron: presentar diagnóstico de TDAH según médicos derivantes, cumplir con los criterios diagnósticos para el TDAH según el DSM-5 (APA, 2013), presentar valores por encima del punto de corte esperado en la escala específica de TDAH, y un nivel intelectual $(\mathrm{Cl})$ promedio. Los criterios de inclusión para la muestra control fueron: no cumplir el criterio diagnóstico para el TDAH, presentar valores por debajo del punto de corte en la escala específica de TDAH y un $\mathrm{Cl}$ promedio. Se excluyeron niños con antecedentes de enfermedades neurológicas o psiquiátricas y trastornos del aprendizaje.

\section{Instrumentos}

Para la confirmación del diagnóstico médico de TDAH se utilizaron los criterios del DSM-5 (APA, 2013) y la escala estandarizada de Swanson, Nolan y Pelham (SNAP IV), en su versión en español validada en Argentina (Grañana et al., 2011). Esta fue administrada tanto a los padres como a los docentes de los niños. Asimismo, para descartar un déficit del nivel intelectual se administró el test de inteligencia breve de Reynolds (RIST) (Reynolds, \& Kamphaus, 2009).

Para evaluar la TDBR se administró The Game of Dice Task (GDT) (Brand et al., 2005), una tarea informatizada en la que un dado se tira 18 veces y la persona debe apostar para ganar puntos. Se puede apostar a un único resultado (p. ej. el número 6) que otorga una recompensa de 1.000; a una combinación de dos resultados (e.g., los números 50 6), que otorgan una recompensa de 500; o a una combinación de tres o cuatro resultados con las respectivas recompensas de 200 y 100 . Si no sale el número elegido pierde la misma cantidad de puntos que la recompensa (1.000, 500, 200 o 100). Las opciones con tres y cuatro dados se consideran las opciones de seguridad, mientras que las opciones de uno o dos dados son consideradas riesgosas. La información sobre las cantidades de ganancias y pérdidas y sus probabilidades de ganar se proporcionan explícitamente desde el inicio de la tarea, de modo que el participante puede predecirla mediante el cálculo. Los puntajes que se consideran son: la cantidad de opciones de uno (arriesgadas), dos (desventajosas), tres (no arriesgadas), o cuatro dados (ventajosas) elegidas, el número de opciones seguras y el número de opciones de riesgo y la ganancia.

Para evaluar la TDBI se administró The Hungry Donkey Task (HDT), de Crone y Van der Molen (2004). Es una prueba informatizada adaptada de la prueba lowa Gambling Task (IGT) de Bechara et al. (1994), diseñada para medir la capacidad de tomar decisiones afectivas o bajo incertidumbre en niños y adolescentes. En la pantalla se presentan cuatro puertas (A, B, C y D) y un burro sentado delante de las puertas. Los participantes deben recolectar la mayor cantidad de manzanas verdes para el burro seleccionando una de las cuatro puertas. Por cada apertura de una puerta, la persona gana o pierde manzanas. Las puertas $C$ y $D$ son consideradas ventajosas o seguras, ya que proveen recompensas inmediatas más pequeñas, pero conducen a rendimientos más grandes a largo plazo ya que las pérdidas son más pequeñas; mientras que las puertas A y B son consideradas desventajosas o riesgosas, ya que otorgan grandes recompensas inmediatas, pero conducen a rendimientos menores a largo plazo porque las pérdidas son mayores. La información sobre las recompensas y pérdidas no es suministrada a los participantes, sino que puede ser inferida. Los puntajes del HDT son: cantidad de opciones seguras (puertas $C$ y D) y cantidad de opciones riesgosas (puertas $A$ y $B$ ) elegidas con sus respectivos tiempos de reacción (TR) y la ganancia. La prueba consta de dos bloques de 50 ensayos con un total de 100 ensayos presentados.

Al finalizar cada prueba se utilizó la prueba de conciencia o autopercepción, que consiste en la realización de cuatro preguntas para evaluar el reconocimiento y la comprensión de las opciones ventajosas y desventajosas. Los participantes deben indicar cuál consideran que es la mejor opción para elegir y justificar su elección, y después, cuál consideran que es la peor opción para elegir y justificar su elección.

\section{Procedimiento}

En el marco de convenios con dos instituciones de salud, y a partir de datos proporcionados por el médico derivador, se establecieron los contactos con los niños y adolescentes con TDAH y sus padres, quienes fueron citados en las instalaciones del instituto para realizar la evaluación individual. Antes del encuentro se les solicitó a los padres que, durante el periodo de evaluación y durante las 24 horas previas a esta, los niños y adolescentes que se encontraban en tratamiento farmacológico, no recibieran medicación. Por otro lado, se procedió al contacto y evaluación de los niños de la muestra control y se administraron los cuestionarios a sus padres y docentes. La participación fue voluntaria y sujeta al asentimiento de los niños y al consentimiento informa- 
do de los padres y adolescentes. Durante el desarrollo del trabajo se respetaron los principios éticos de la investigación con seres humanos de la Declaración de Helsinki, procurándose las condiciones necesarias para proteger la confidencialidad y actuar en beneficio de los participantes.

\section{Análisis estadístico}

Para alcanzar los objetivos propuestos se realizaron análisis estadísticos descriptivos. Asimismo, se sometieron los datos a la prueba no paramétrica $U$ de Mann Whitney para comparación de medias, para dos muestras independientes. Se utilizó esta prueba dado el tamaño de la muestra, ya que es una alternativa a la prueba t sobre diferencia de medias cuando no se cumplen los supuestos en los que se basa la prueba t (normalidad y homocedasticidad) (Pardo, \& Ruiz, 2002). Además, se calculó el tamaño del efecto de la diferencia, para lo cual se utilizó la clasificación por rangos de Cohen, que indica que el tamaño del efecto puede ser grande (mayor a 0.8), mediano (cercano a 0.5) o menor (menor a 0.2) (Quezada, 2007).

\section{Resultados}

En la tabla 1 se analiza el desempeño de los grupos con y sin TDAH en función de los grupos etarios. Tal como se observa los niños con diagnóstico de TDAH presentaron un desempeño similar a los niños de la muestra control en ambos tipos de TD.

Por su parte, los adolescentes con diagnóstico de TDAH presentaron un mayor nivel de riesgo en el desempeño en TD. Respecto a la TDBI, presentaron una mayor cantidad de respuestas riesgosas y una menor cantidad de respuestas seguras aunque sin diferencias estadísticamente significativas. En relación con la TDBR, también presentaron una mayor cantidad de respuestas riesgosas y una menor cantidad de respuestas seguras siendo estas diferencias estadísticamente significativas con un tamaño del efecto mediano. En cuanto a los tiempos de reacción, los adolescentes de la muestra clínica presentaron mayores tiempos que los pares sin TDAH en ambos tipos de TD, observándose diferencias significativas en elecciones riesgosas de TDBR con un tamaño del efecto mediano. Y en lo que respecta a la ganancia, en ambos tipos de TD si bien la muestra control alcanzó puntajes más altos, estas diferencias no fueron estadísticamente significativas.

Cuando se analizan las diferencias entre niños y adolescentes intragrupo, en el grupo control se observa que en TDBI no hay desempeños diferentes entre ambos grupos etarios, excepto en los TR de las respuestas de la puerta $\mathrm{B}(p=.02$, U de Mann Whitney $=.73)$ y de las respuestas seguras $(p=.01, \mathrm{U}=67)$ en los cuales los adolescentes responden más rápido que los niños. Y en TDBR los adolescentes logran alcanzar mayores ganancias $(p=.01, U=69.5)$, presentando menor cantidad de elecciones de riesgo $(p=.01, \mathrm{U}=70)$ y mayor cantidad de elecciones seguras $(p=.01, \mathrm{U}=67)$ que los niños.

$Y$ en el grupo con TDAH se observa que en TDBI los adolescentes alcanzan mejores ganancias que los niños $(p=.03$, $\mathrm{U}=74$ ), con mayor cantidad de respuestas de riesgo $(p=.03, \mathrm{U}=74)$ y menor cantidad de respuestas seguras $(p=.03, U=74)$ que los niños. También presentan menores TR, siendo esta diferencia estadísticamente significativa solo para TR de la elección de la puerta $\mathrm{B}(p=.04, \mathrm{U}=78)$.

$Y$ respecto a TDBR se puede observar que los adolescen-

Tabla 1 Estadísticos descriptivos, prueba U-Mann-Whitney (U) y tamaño del efecto (d' de Cohen) para TDBI y TDBR discriminados en función del grupo etario y la pertenencia al grupo

\begin{tabular}{|c|c|c|c|c|c|c|c|c|c|c|}
\hline & & & \multicolumn{5}{|c|}{ Niños $(n=15)$} & \multicolumn{3}{|c|}{ Adolescentes $(n=20)$} \\
\hline & & Grupo & $M(D E)$ & $\mathrm{p}$ & $U$ & $d^{\prime}$ & $M(D E)$ & p & $U$ & $d^{\prime}$ \\
\hline \multirow{16}{*}{ TDBI } & \multirow{2}{*}{ Ganancia } & Control & $304.62(11.62)$ & .43 & 80.5 & & $305.62(15.41)$ & .05 & 118 & \\
\hline & & TDAH & 301.33 (17.34) & & & & $318.11(28.21)$ & & & \\
\hline & \multirow{2}{*}{$\mathrm{N}$ opción $\mathrm{A}$} & Control & $19.69(4.29)$ & .14 & 66 & & $18.95(6.40)$ & .30 & 152.5 & \\
\hline & & TDAH & $17.40(4.24)$ & & & & $17.94(7.17)$ & & & \\
\hline & \multirow{2}{*}{ TR opción A } & Control & 1563.66 (577.64) & .22 & 71 & & 1.226 .01 (385.37) & .09 & 129 & \\
\hline & & TDAH & 1895.21 (993.99) & & & & $1552.53(602.21)$ & & & \\
\hline & \multirow{2}{*}{$N$ opción B } & Control & $32.62(6.64)$ & .73 & 90 & & $33.86(10.08)$ & .05 & 118 & \\
\hline & & TDAH & $33.27(9.05)$ & & & & 41.11(13.70) & & & \\
\hline & \multirow{2}{*}{ TR opción $B$} & Control & 1578.97 (712.21) & .42 & 80 & & $1120.29(481.48)$ & .11 & 133 & \\
\hline & & TDAH & 2019.18 (1118.66) & & & & $1359.30(487.10)$ & & & \\
\hline & \multirow{2}{*}{$\mathrm{N}$ opción C } & Control & $25.15(3.34)$ & .28 & 74 & & $22.95(5.41)$ & $.02^{*}$ & 106.5 & .77 \\
\hline & & TDAH & $22.67(5.192)$ & & & & $17.67(7.69)$ & & & \\
\hline & \multirow{2}{*}{ TR opción C } & Control & 1653.46 (477.26) & .84 & 93 & & 1196.09 (471.97) & .12 & 134 & \\
\hline & & TDAH & 1955.89 (1116.58) & & & & $1680.68(1092.31)$ & & & \\
\hline & \multirow{2}{*}{$N$ opción D } & Control & $22.54(4.86)$ & .12 & 64 & & $24.24(7.65)$ & .28 & 151 & \\
\hline & & TDAH & $26.67(9.13)$ & & & & 23.28 (12.69) & & & \\
\hline
\end{tabular}


Tabla 1 Estadísticos descriptivos, prueba U-Mann-Whitney (U) y tamaño del efecto (d' de Cohen) para TDBI y TDBR discriminados en función del grupo etario y la pertenencia al grupo

\begin{tabular}{|c|c|c|c|c|c|c|c|c|c|c|}
\hline & & \multirow[b]{2}{*}{ Grupo } & \multicolumn{5}{|c|}{ Niños $(n=15)$} & \multicolumn{3}{|c|}{ Adolescentes $(n=20)$} \\
\hline & & & $M(D E)$ & $\mathrm{p}$ & $\mathbf{U}$ & $d^{\prime}$ & $M(D E)$ & $\mathrm{p}$ & U & $d^{\prime}$ \\
\hline \multirow{10}{*}{ TDBI } & \multirow{2}{*}{ TR opción D } & Control & $1550.75(634.09)$ & .98 & 97 & & 1181.99 (591.22) & .38 & 158 & \\
\hline & & TDAH & $1794.06(1260.54)$ & & & & $1386.60(745.18)$ & & & \\
\hline & \multirow{2}{*}{$\begin{array}{l}\text { N Respuestas } \\
\text { seguras }\end{array}$} & Control & $47.69(5.81)$ & .43 & 80.5 & & 47.19 (7.70) & .05 & 118 & \\
\hline & & TDAH & $49.33(8.67)$ & & & & $40.94(14.11)$ & & & \\
\hline & \multirow{2}{*}{$\begin{array}{l}\text { TR Respuestas } \\
\text { seguras }\end{array}$} & Control & 1602.12 (519.11) & .91 & 95 & & 1189.04 (490.29) & .13 & 135 & \\
\hline & & TDAH & $1874.98(1149.49)$ & & & & 1533.64 (819.58) & & & \\
\hline & \multirow{2}{*}{$\begin{array}{l}\text { N Respuestas de } \\
\text { riesgo }\end{array}$} & Control & $52.31(5.81)$ & .43 & 80.5 & & $52.81(7.70)$ & .05 & 118 & \\
\hline & & TDAH & $50.67(8.67)$ & & & & $59.06(14.11)$ & & & \\
\hline & \multirow{2}{*}{$\begin{array}{l}\text { TR Respuestas } \\
\text { de riesgo }\end{array}$} & Control & $785.66(308.46)$ & .28 & 74 & & 586.57 (194.67) & .06 & 121 & \\
\hline & & TDAH & $978.60(494.68)$ & & & & $727.96(218.23)$ & & & \\
\hline \multirow{26}{*}{ TDBR } & \multirow{2}{*}{ N 1 dado } & Control & $5.07(2.53)$ & .08 & 60.5 & & $2.00(3.07)$ & $.01^{*}$ & 107 & .64 \\
\hline & & TDAH & $7.21(4.17)$ & & & & $4.42(4.34)$ & & & \\
\hline & \multirow{2}{*}{ TR 1 dado } & Control & $4990(3260)$ & .35 & 77.5 & & $3.06(5.15)$ & $.01^{*}$ & 102 & .55 \\
\hline & & TDAH & 7540 (7190) & & & & $6.96(8.50)$ & & & \\
\hline & \multirow{2}{*}{ N 2 dados } & Control & 3.29 (1.77) & .56 & 85.5 & & $2.33(2.06)$ & .11 & 142.5 & \\
\hline & & TDAH & $3.50(3.67)$ & & & & $3.32(2.36)$ & & & \\
\hline & \multirow{2}{*}{ TR 2 dados } & Control & $4210(2760)$ & .35 & 77.5 & & $4.15(4.78)$ & $.03^{*}$ & 118 & .43 \\
\hline & & TDAH & $5840(4200)$ & & & & $6.18(4.57)$ & & & \\
\hline & \multirow{2}{*}{ N 3 dados } & Control & 3.79 (1.63) & $.00^{* *}$ & 38 & 1.18 & $4.86(3.05)$ & .20 & 153 & \\
\hline & & TDAH & $1.93(1.49)$ & & & & $4.16(3.45)$ & & & \\
\hline & \multirow{2}{*}{ TR 3 dados } & Control & $4470(2170)$ & .84 & 93.5 & & $3.65(2.65)$ & .80 & 190 & \\
\hline & & TDAH & $4480(2910)$ & & & & $3.87(2.75)$ & & & \\
\hline & \multirow{2}{*}{ N 4 dados } & Control & $5.86(3.82)$ & .49 & 83 & & $8.81(4.58)$ & .07 & 132 & \\
\hline & & TDAH & $5.36(4.89)$ & & & & $6.11(4.23)$ & & & \\
\hline & \multirow{2}{*}{ TR 4 dados } & Control & $4690(3980)$ & .17 & 68 & & $3.47(2.32)$ & .76 & 188 & \\
\hline & & TDAH & $4830(2210)$ & & & & $3.25(1.95)$ & & & \\
\hline & \multirow{2}{*}{$\begin{array}{l}\mathrm{N} \text { Respuestas de } \\
\text { riesgo }\end{array}$} & Control & $8.36(3.61)$ & .16 & 67.5 & & $4.33(4.90)$ & $.01^{*}$ & 99.5 & .71 \\
\hline & & TDAH & $10.71(5.14)$ & & & & $7.74(4.62)$ & & & \\
\hline & \multirow{2}{*}{$\begin{array}{l}\text { TR Respuestas } \\
\text { de riesgo }\end{array}$} & Control & $4700(2700)$ & .12 & 64.5 & & $4.98(5.97)$ & $.02^{*}$ & 110.5 & .30 \\
\hline & & TDAH & $7540(7110)$ & & & & $6.67(5.03)$ & & & \\
\hline & \multirow{2}{*}{$\begin{array}{l}\text { N Respuestas } \\
\text { seguras }\end{array}$} & Control & $9.64(3.61)$ & .16 & 67.5 & & $13.67(4.90)$ & $.01^{*}$ & 99.5 & .71 \\
\hline & & TDAH & $7.29(5.14)$ & & & & $10.26(4.62)$ & & & \\
\hline & \multirow{2}{*}{$\begin{array}{l}\text { TR Respuestas } \\
\text { seguras }\end{array}$} & Control & $4.82(3.05)$ & .08 & 59.5 & & $4.00(2.00)$ & .84 & 192 & \\
\hline & & TDAH & $5.55(1.55)$ & & & & $3.65(1.75)$ & & & \\
\hline & \multirow{2}{*}{ Ganancia } & Control & $-3642.86(2979.34)$ & .80 & 92.5 & & $-485.71(3191.75)$ & .05 & 127 & \\
\hline & & TDAH & $-3757.14(3481.98)$ & & & & $-1605.26(3696.32)$ & & & \\
\hline
\end{tabular}

Referencias: TDBI: toma de decisiones bajo incertidumbre; TDBR: toma de decisiones bajo riesgo; N: cantidad de elecciones; TR: tiempos de reacción.

${ }^{*}: p<.05 ;{ }^{* *}: p<.01$. 
tes alcanzan mejores ganancias que los niños $(p=.03, U=74)$, siendo que los niños eligen mayor cantidad de opciones de riesgosas de un dado $(p=.04, U=76.5)$ y menor cantidad de elecciones seguras de tres dados $(p=.03, \mathrm{U}=75.5)$. Respecto a los TR, los adolescentes demoran menos en seleccionar las opciones seguras $(p=.00, \mathrm{U}=50.5)$.

Respecto a la prueba de conciencia o autopercepción (tabla 2) la muestra de niños con diagnóstico de TDAH presentó un menor reconocimiento y comprensión de cuáles eran las opciones ventajosas y desventajosas en TDBR con diferencias estadísticamente significativas con tamaño de efecto grande, mientras que no se encontraron diferencias con el grupo control en la TDBI.

Los adolescentes de la muestra clínica mostraron un menor reconocimiento y comprensión de las opciones ventajosas y desventajosas, aunque estas diferencias solo fueron estadísticamente significativas en la justificación de las opciones ventajosas y desventajosas de la TDBI con un tamaño del efecto mediano y grande respectivamente.

En el gráfico 1 pueden observarse las elecciones de riesgo realizadas por los niños y los adolescentes de ambos grupos, con y sin TDAH, en las dos partes de las pruebas de TDBI. La primera parte de la prueba corresponde a las elecciones realizadas en los primeros 50 intentos, donde no se hallaron diferencias significativas entre ambos grupos en ambas edades. Y en la segunda parte se observa que en ambas muestras aumentó el número de elecciones de riesgo, sin diferencias significativas en los niños y con diferencias significativas en los adolescentes ( $p=.02 ; U=108)$.

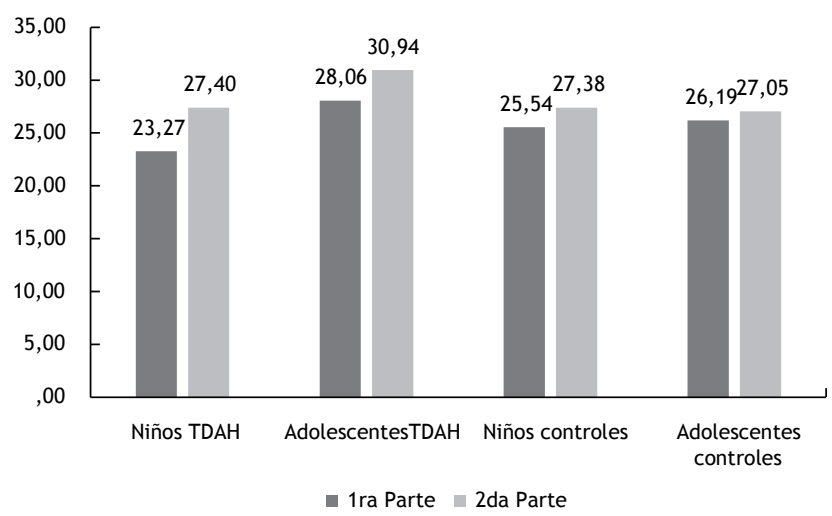

Gráfico 1. Elecciones de riesgo realizadas por los niños y adolescentes de ambos grupos en la primera y segunda parte de la prueba de TDBI
Respecto a la prueba de TDBR (gráfico 2), en la primera parte correspondiente a las elecciones realizadas en los primeros nueve intentos, se observa que tanto los niños como los adolescentes de la muestra clínica realizaron mayor cantidad de elecciones de riesgo con diferencias significativas (niños $p=.04, U=54$; adolescentes $p=.01, U=111$ ) respecto a los controles. En la segunda mitad de la prueba de TDBR también se observa que tanto los niños como los adolescentes de la muestra clínica realizaron mayor cantidad de elecciones de riesgo con diferencias significativas en adolescentes (adolescentes $p=.03, U=122.5$ ) respecto a los controles. $Y$ se puede observar que en los niños del grupo con TDAH disminuye el número de elecciones riesgosas, contrario a lo que sucede con el grupo control, en los cuales aumentan.

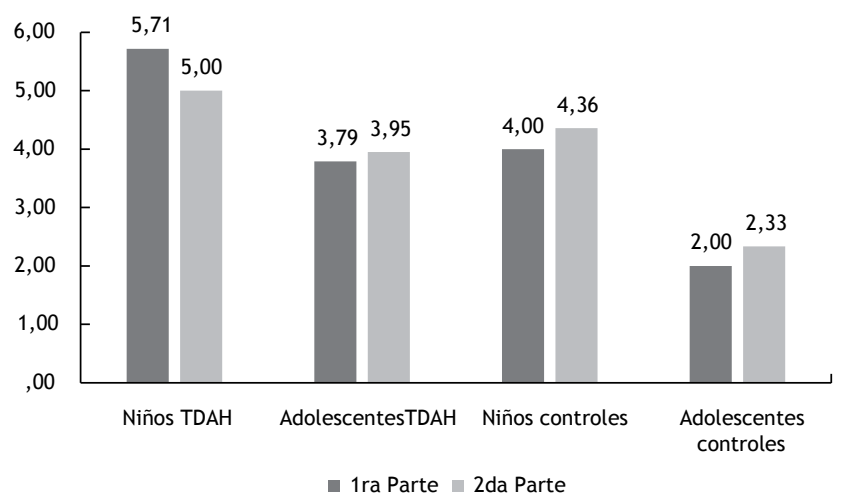

Gráfico 2. Elecciones de riesgo realizadas por los niños y adolescentes de ambos grupos en la primera y segunda parte de la prueba de TDBR

\section{Discusión}

Las investigaciones sobre la TD en niños y adolescentes con TDAH han reportado resultados controvertidos, mientras que algunas coinciden en señalar que son propensos a presentar dificultades en la toma de decisiones otros sugieren no haber encontrado diferencias (Rubiales et al., 2017).

Los niños con TDAH presentaron un comportamiento similar a los controles en ambos tipos de TD, tanto en aquellas situaciones en las que la información de los posibles resultados y consecuencias potenciales no es anticipada y debe inferirse, como cuando las reglas son explicitadas desde un comienzo. Estos resultados se corresponden con las

Tabla 2. Estadísticos descriptivos, resultados de la prueba U-Mann-Whitney y tamaño del efecto para la prueba de conciencia en TDBI y TDBR discriminados en función del grupo etario y la pertenencia al grupo

\begin{tabular}{|c|c|c|c|c|c|c|c|c|c|c|c|}
\hline & & \multicolumn{2}{|c|}{ Niños $(n=15)$} & \multirow[t]{2}{*}{$\mathrm{p}$} & \multirow[t]{2}{*}{ U } & \multirow[t]{2}{*}{$d^{\prime}$} & \multicolumn{2}{|c|}{ Adolescentes $(n=20)$} & \multirow[t]{2}{*}{$\mathrm{p}$} & \multirow[t]{2}{*}{$U$} & \multirow[t]{2}{*}{$d^{\prime}$} \\
\hline & & $\begin{array}{l}\text { Control } \\
M(D E)\end{array}$ & $\begin{array}{c}\text { TDAH } \\
M(D E)\end{array}$ & & & & $\begin{array}{l}\text { Control } \\
M(D E)\end{array}$ & $\begin{array}{l}\text { TDAH } \\
M(D E)\end{array}$ & & & \\
\hline \multirow{4}{*}{ TDBI } & Mejor opción & $.23(.44)$ & $.20(.41)$ & .84 & 94.5 & & $.33(.48)$ & $.17(.38)$ & .24 & 157.5 & \\
\hline & Porque mejor & $.23(.44)$ & $.20(.41)$ & .84 & 94.5 & & $.33(.48)$ & $.06(.24)$ & $.03^{*}$ & 136.5 & .37 \\
\hline & Peor opción & $.23(.44)$ & $.40(.51)$ & .34 & 81.0 & & $.67(.48)$ & $.39(.50)$ & .08 & 136.5 & \\
\hline & Porque peor & $.23(.44)$ & $.27(.46)$ & .83 & 94.0 & & $.67(.48)$ & $.22(.43)$ & $.00^{* *}$ & 105.0 & .98 \\
\hline \multirow{4}{*}{ TDBR } & Mejor opción & $1.00(.00)$ & $.42(.52)$ & $.00^{* *}$ & 35.0 & 1.57 & $.86(.36)$ & $.67(.49)$ & .18 & 127.5 & \\
\hline & Porque mejor & $.79(.43)$ & $.25(.45)$ & $.00^{* *}$ & 39.0 & 1.22 & $.76(.44)$ & $.67(.49)$ & .53 & 142.5 & \\
\hline & Peor opción & $.93(.27)$ & $.50(.52)$ & $.01^{*}$ & 48.0 & 1.03 & $.90(.30)$ & $.73(.46)$ & .18 & 130.5 & \\
\hline & Porque peor & $.64(.50)$ & $.08(.29)$ & $.00^{* *}$ & 37.0 & 1.37 & $.76(.44)$ & $.53(.52)$ & .15 & 121.5 & \\
\hline
\end{tabular}

${ }^{*}: p<.05 ;{ }^{* *}: p<.01$. 
evidencias aportadas por los estudios que indican no haber encontrado diferencias en el desempeño de TD en este grupo etario (Antonini et al., 2015; Geurts et al., 2006; Hovik, \& Hansen 2015; Lambek et al., 2011; Masunami et al., 2009; Skogli et al., 2013). Los adolescentes presentaron un desempeño con tendencia más riesgosa en TDBI, aunque lograron alcanzar puntajes finales favorables. Por el contrario, en TDBR los adolescentes con diagnóstico de TDAH presentaron un comportamiento más riesgoso, aunque obtuvieron un mejor desempeño que los niños de la población clínica, lo cual contrasta con lo que sugieren Bernal et al. (2015) al señalar que en los adolescentes con TDAH no se observa una mejora gradual en el desempeño de la TD. Además, resulta interesante observar que de igual modo que lo indicado en TDBI, en TDBR a pesar de haber asumido mayores niveles de riesgo, en lo que respecta a los resultados obtenidos en términos de ganancia presentaron un rendimiento semejante a la población control. De modo que los adolescentes de la muestra clínica parecen utilizar diferentes estrategias para alcanzar el objetivo planteado. El uso diferencial de estrategias podría estar relacionado con la búsqueda de recompensas inmediatas y una menor capacidad para anticipar consecuencias a largo plazo, tal como indican Toplak et al. (2005). Además, los adolescentes con TDAH demoraron más tiempo en las elecciones riesgosas de la TDBR, en contraste con los estudios que señalan que lo hacen más rápidamente y de manera impulsiva (Kroyzer et al., 2014).

Por otra parte, el grado de reconocimiento y comprensión de las opciones ventajosas y desventajosas fue similar en niños de la muestra clínica y control en la TDBI, sin embargo, los controles parecerían beneficiarse cuando la situación que se les ofrece es más estructurada y disponen de reglas explícitas para elegir como ocurre en la TDBR. La dificultad para reconocer cuáles son las mejores y peores opciones, así como para argumentar dicha elección, parecería estar relacionada con la presencia de respuestas impulsivas o automáticas. Sin embargo, un análisis más exhaustivo sugiere que estos resultados parecen reflejar un enfoque particular en el procesamiento que realizan para efectuar la decisión, centrado en la magnitud de la recompensa o la pérdida, y en menor medida en el cálculo de probabilidad asociada a una opción, la cual parece no ser considerada generalmente. Por otra parte, los adolescentes del grupo clínico mostraron una menor capacidad de argumentación o justificación de las opciones ventajosas y desventajosas en la TDBI aunque una capacidad similar en el grado de conciencia en la TDBR, de modo que en este momento evolutivo, sí parecen beneficiarse de la disponibilidad de información explícita acerca de los posibles resultados y consecuencias aunque conserven más dificultades cuando se deben inferir las reglas implícitas a lo largo del proceso de TD.

De lo anterior se desprende que las características de los adolescentes con TDAH parecen ser más compatibles con la presencia de un estilo diferente en la TD, que con una propensión a la TD desfavorable como sugieren diversos estudios (Kroyzer et al., 2014; Scheres et al., 2004; Skogli et al., 2013, Skogli et al., 2014), dado que un comportamiento más riesgoso no parece ser sinónimo de TD desfavorable. Como se mencionó, si bien presentaron mayor cantidad de respuestas riesgosas, no se encontraron diferencias en cuanto a los resultados obtenidos. De este modo, los adolescentes con TDAH no presentarían una alteración o déficit en el proceso de TD, lo que amplía la visión desde una perspectiva positiva, y posibilita un mejor conocimiento de la entidad, lo que a su vez implicaría cambios para la práctica clínica y la planificación de un tratamiento neuropsicológico integral adaptado a las necesidades y problemáticas de cada niño.

Es importante mencionar que una de las limitaciones de este trabajo está relacionada con el tamaño de la muestra, debido a los inconvenientes que se presentan habitualmente para evaluar pacientes diagnosticados con alguna patología y que cumplan con todos los criterios de inclusión que se requieren en un diseño de investigación. Otra limitación podría vincularse a que la prueba utilizada en la presente investigación para evaluar la TDBI puede generar en los niños menos motivación ya que deben decidir por un "burro", que es quien finalmente recibe las recompensas y no por sí mismo, lo cual puede ocasionar una menor implicación en la tarea (Skogli et al., 2013).

Una línea de trabajo interesante para continuar desarrollando está vinculada con analizar la relación entre TD, funciones ejecutivas y variables emocionales. Asimismo, otra línea de interés es el desarrollo de instrumentos de evaluación de TD que aporten validez ecológica referida a que el desempeño en la prueba posibilite inferir la capacidad funcional del niño en sus actividades cotidianas.

\section{Referencias}

Antonini, T. N., Becker, S. P., Tamm, L., \& Epstein, J. N. (2015). Hot and cool executive functions in children with attention-deficit/ hyperactivity disorder and comorbid oppositional defiant disorder. Journal of the International Neuropsychological Society, 21(8), 584-595. http://dx.doi.org/10.1017/S1355617715000752

Bechara, A. (2004). The role of emotion in decision-making: Evidence from neurological patients with orbitofrontal damage. Brain and Cognition, 55(1), 30-40. http://dx.doi.org/10.1016/j. bandc.2003.04.001

Bechara, A., Damasio, A. R., Damasio, H., \& Anderson, S. W. (1994). Insensitivity to future consequences following damage to human prefrontal cortex. Cognition, 50(1-3), 7-15. http://dx.doi. org/10.1016/0010-0277(94) 90018-3.

Bernal, P., Montaña, J., Acosta, R., \& Rojas, Y. (2015). Performance of children and adolescents from a school of the city of Sogamoso on a decision-making test. Open Journal of Pediatrics, 5, 339-347. http://dx.doi.org/10.4236/ojped.2015.54051

Brand, M., Fujiwara, E., Borsutzky, S., Kalbe, E., Kessler, J., \& Markowitsch, H. J. (2005). Decision-making deficits of Korsakoff patients in a new gambling task with explicit rules: Associations with executive functions. Neuropsychology, 19(3), 267. http:// dx.doi.org/10.1037/0894-4105.19.3.267

Brand, M., Heinze, K., Labudda, K., \& Markowitsch, H. J. (2008). The role of strategies in deciding advantageously in ambiguous and risky situations. Cognitive Processing, 9(3), 159-173. http:// dx.doi.org/10.1007/s10339-008-0204-4

Brand, M., Labudda, K., \& Markowitsch, H. J. (2006). Neuropsychological correlates of decision-making in ambiguous and risky situations. Neural Networks, 19, 1266-1276. http://dx.doi.org/10. 1016/j.neunet.2006.03.001

Crone, E. A., \& Van der Molen, M. W. (2004). Developmental changes in real life decision making: performance on a gambling task previously shown to depend on the ventromedial prefrontal cortex. Developmental Neuropsychology, 25(3), 251-279. http://dx.doi.org/10.1207/s15326942dn2503_2

American Psychiatric Association. (2013). Statistical manual of mental disorders, fifth edition (DSM-5). Washington: American Psychiatric Association.

Drechsler, R., Rizzo, P., \& Steinhausen, H. C. (2010). Decision making with uncertain reinforcement in children with attention deficit/hyperactivity disorder (ADHD). Child Neuropsychology, 16(2), 145-161. http://dx.doi.org/10.1080/09297040903190774 
Garon, N., Moore, C., \& Waschbusch, D. A. (2006). Decision making in children with ADHD only, ADHD-anxious/depressed, and control children using a child version of the lowa Gambling Task. Journal of Attention Disorders, 9(4), 607-619. http://dx.doi. org/10.1177/1087054705284501

Geurts, H. M., Van der Oord, S., \& Crone, E. A. (2006). Hot and cool aspects of cognitive control in children with ADHD: Decision-making and inhibition. Journal of Abnormal Child Psychology, 34(6), 811-822.

Grañana, N. (2017). Clínica de trastornos de atención y memoria. En Fejerman, N., \& Grañana, N. Neuropsicología infantil (pp. 441-470). Buenos Aires: Paidós. http://dx.doi.org/10.5579/rnl. 2011.0076

Grañana, N., Richaudeau, A., Gorriti, C. R., O’Flaherty, M., Scotti, M. E., Sixto, L., Allegri, R., \& Fejerman, N. (2011). Evaluación de déficit de atención con hiperactividad: la escala SNAP IV adaptada a la Argentina. Revista Panamericana de Salud Pública, 29, 344-349.

Groen, Y., Gaastra, G. F., Lewis-Evans, B., \& Tucha, O. (2013). Risky behavior in gambling tasks in individuals with ADHD-a systematic literature review. PLoS One, 8(9), e74909. http://dx.doi. org/10.1371/journal.pone.0074909

Hobson, C. W., Scott, S., \& Rubia, K. (2011). Investigation of cool and hot executive function in ODD/CD independently of ADHD. Journal of Child Psychology and Psychiatry, 52(10), 1035-1043. http://dx.doi.org/10.1111/j.1469-7610.2011.02454.x

Hovik, S., \& Hanssen, G. S. (2015). The impact of network management and complexity on multi-level coordination. Public Administration, 93(2), 506-523. http://dx.doi.org/10.1111/padm. 12135

Hovik, K. T., Plessen, K. J., Cavanna, A. E., Skogli, E. W., Andersen, P. N., \& Oie, M. (2015). Cognition, emotion and behavior in children with Tourette's syndrome and children with ADHD-combined subtype-A two-year follow-up study. PloS one, 10, 1-22.

Humphreys, K. L., Tottenham, N., \& Lee, S. S. (2018). Risky decision-making in children with and without ADHD: a prospective study. Child Neuropsychology, 24(2), 261-276.

Kahneman, D., \& Tversky, A. (1984). Choices, values, and frames. American Psychologist, 39(4), 341-350.

Kroyzer, N., Gross-Tsur, V., \& Pollak, Y. (2014). Risk taking in adolescents with attention deficit hyperactivity disorder on a probabilistic choice task. The Journal of Nervous and Mental Disease, 202(3), 247-252. http://dx.doi.org/10.1097/ NMD. 0000000000000100

Lambek, R., Tannock, R., Dalsgaard, S., Trillingsgaard, A., Damm, D., \& Thomsen, P. H. (2011). Executive dysfunction in schoolage children with ADHD. Journal of Attention Disorders, 15(8), 646-655. http://dx.doi.org/10.1177/1087054710370935

Liebherr, M., Schiebener, J., Averbeck, H., \& Brand, M. (2017). Decision making under ambiguity and objective risk in higher age-A review on cognitive and emotional contributions. Frontiers in Psychology, 8, 2128.

Mariño, M. C., Ageitos, A. G., Álvarez, J. A., Del Río Garma, M., Cendón, C. G., Castaño, A. G., \& Nieto, J. P. (2018). Prevalencia de trastornos del neurodesarrollo, comportamiento y aprendizaje en atención primaria. Anales de Pediatría, 89(3), 153-161. http://dx.doi.org/10.1016/j.anpedi.2017.10.007

Masunami, T., Okazaki, S., \& Maekawa, H. (2009). Decision-making patterns and sensitivity to reward and punishment in children with attention-deficit hyperactivity disorder. International Journal of Psychophysiology, 72(3), 283-288. http://dx.doi.org/10.1016/j.ijpsycho.2009.01.007

Montero, I., \& León, O. G. (2007). A guide for naming research studies in psychology. International Journal of Clinical and Health Psychology, 7(3).
Oie, M., Skogli, E. W., Andersen, P. N., Hovik, K. T., \& Hugdahl, K. (2014). Differences in cognitive control in children and adolescents with combined and inattentive subtypes of ADHD. Child Neuropsychology, 20(1), 38-48. http://dx.doi.org/10.1080/0929 7049.2012.741224

Pardo, A., \& Ruiz, M. A. (2002). Spss 11: guía para el análisis de datos. México: McGraw-Hill.

Patros, C. H., Alderson, R. M., Lea, S. E., Tarle, S. J., Kasper, L. J., \& Hudec, K. L. (2015). Visuospatial working memory underlies choice-impulsivity in boys with attention-deficit/hyperactivity disorder. Research in Developmental Disabilities, 38, 134-144. http://dx.doi.org/10.1016/j.ridd.2014.12.016

Quezada, C. (2007). Potencia estadística, sensibilidad y tamaño de efecto: ¿un nuevo canon para la investigación? Onomázein: Revista de Lingüística, Filología y Traducción de la Pontificia Universidad Católica de Chile (16), 159-170.

Reyna, V., \& Farley, F. (2006). Risk and rationality in adolescent decision making: Implications for theory practice, and public policy. Psychological Science in the Public Interest, 7, 1-44. http:// dx.doi.org/10.1111/j.1529-1006.2006.00026.x

Reynolds, C. R. \& Kamphaus, R. W. (2009). RIAS escalas de inteligencia de Reynolds y RIST test de inteligencia breve de Reynolds. Madrid: TEA.

Rubiales, J., Urquijo, S., Said, A., \& Macbeth, G. (2017). Proceso de toma de decisiones en niños y adolescentes con TDAH: revisión sistemática. Revista de Psicopatología y Psicología Clínica, 22(2), 139-155. http://dx.doi.org/10.5944/rppc.vol.22. num.2.2017.17688

Scheres, A., Dijkstra, M., Ainslie, E., Balkan, J., Reynolds, B., Sonuga-Barke, E., \& Castellanos, F. X. (2006). Temporal and probabilistic discounting of rewards in children and adolescents: effects of age and ADHD symptoms. Neuropsychologia, 44(11), 2092-2103. http://dx.doi.org/10.1016/j.neuropsychologia.2005. 10.012

Schiebener, J., \& Brand, M. (2015). Decision making under objective risk conditions-a review of cognitive and emotional correlates, strategies, feedback processing, and external influences. Neuropsychology Review, 25(2), 171-198.

Schiebener, J., \& Brand, M. (2017). Age-related variance in decisions under ambiguity is explained by changes in reasoning, executive functions, and decision-making under risk. Cognition and Emotion, 31(4), 816-824.

Skogli, E. W., Teicher, M. H., Andersen, P. N., Hovik, K. T., \& Oie, M. (2013). ADHD in girls and boys-gender differences in co-existing symptoms and executive function measures. BMC Psychiatry, 13(1), 298. http://dx.doi.org/10.1186/1471-244X-13-298

Sonuga-Barke, E. J. (2002). Psychological heterogeneity in AD/ HD-a dual pathway model of behaviour and cognition. Behavioural Brain Research, 130(1-2), 29-36.

Toplak, M. E., Jain, U., \& Tannock, R. (2005). Executive and motivational processes in adolescents with attention-deficit-hyperactivity disorder (ADHD). Behavioral and Brain Functions, 1(1), 8. http://dx.doi.org/10.1186/1744-9081-1-8

Wilbertz, G., Trueg, A., Sonuga-Barke, E. J., Blechert, J., Philipsen, A., \& Tebartz van Elst, L. (2013). Neural and psychophysiological markers of delay aversion in attention-deficit hyperactivity disorder. Journal of Abnormal Psychology, 122(2), 566. http:// dx.doi.org/10.1037/a0031924

Van Duijvenvoorde A. C. K. (2013). Affective and cognitive decision-making in adolescents. En Van Duijvenvoorde, A. C. K. (ed.), On the art of choosing: Developmental changes and individual differences in decision making under risk (pp. 18-31). Leident: Faculty of Social and Behavioural Sciences.

Willcutt, E. G. (2012). The prevalence of DSM-IV attention-deficit/ hyperactivity disorder: A meta-analytic review. Neurotherapeutics, 9(3), 490-499. http://dx.doi.org/10.1007/s13311-012-0135-8 
Scheres, A., Dijkstra, M., Ainslie, E., Balkan, J., Reynolds, B., Sonuga-Barke, E., \& Castellanos, F. X. (2006). Temporal and probabilistic discounting of rewards in children and adolescents: effects of age and ADHD symptoms. Neuropsychologia, 44(11), 2092-2103. http://dx.doi.org/10.1016/j.neuropsychologia.2005. 10.012

Schiebener, J., \& Brand, M. (2015). Decision making under objective risk conditions-a review of cognitive and emotional correlates, strategies, feedback processing, and external influences. Neuropsychology Review, 25(2), 171-198.

Schiebener, J., \& Brand, M. (2017). Age-related variance in decisions under ambiguity is explained by changes in reasoning, executive functions, and decision-making under risk. Cognition and Emotion, 31(4), 816-824.

Skogli, E. W., Teicher, M. H., Andersen, P. N., Hovik, K. T., \& Oie, M. (2013). ADHD in girls and boys-gender differences in co-existing symptoms and executive function measures. BMC Psychiatry, 13(1), 298. http://dx.doi.org/10.1186/1471-244X-13-298

Sonuga-Barke, E. J. (2002). Psychological heterogeneity in AD/ HD-a dual pathway model of behaviour and cognition. Behavioural Brain Research, 130(1-2), 29-36.
Toplak, M. E., Jain, U., \& Tannock, R. (2005). Executive and motivational processes in adolescents with attention-deficit-hyperactivity disorder (ADHD). Behavioral and Brain Functions, 1(1), 8. http://dx.doi.org/10.1186/1744-9081-1-8

Wilbertz, G., Trueg, A., Sonuga-Barke, E. J., Blechert, J., Philipsen, A., \& Tebartz van Elst, L. (2013). Neural and psychophysiological markers of delay aversion in attention-deficit hyperactivity disorder. Journal of Abnormal Psychology, 122(2), 566. http:// dx.doi.org/10.1037/a0031924

Van Duijvenvoorde A. C. K. (2013). Affective and cognitive decision-making in adolescents. En Van Duijvenvoorde, A. C. K. (ed.), On the art of choosing: Developmental changes and individual differences in decision making under risk (pp. 18-31). Leident: Faculty of Social and Behavioural Sciences.

Willcutt, E. G. (2012). The prevalence of DSM-IV attention-deficit/ hyperactivity disorder: A meta-analytic review. Neurotherapeutics, 9(3), 490-499. http://dx.doi.org/10.1007/s13311-012-0135-8 